\title{
Modelling \& Analysis of Engine Mount System for Vibration Reduction
}

\author{
Vishal Vasant Kadam \\ M.Tech Student, Mechanical Engineering Department \\ Vishwakarma Institute of Technology, Pune, India \\ Prof. Nishant S. Kulkarni \\ Assistant professor, Mechanical Engineering Department \\ Vishwakarma Institute of Technology, Pune, India
}

\begin{abstract}
Vehicle engine mounting system consists of an engine and three to four mounts connected to the vehicle structure. The behaviour of mounting system depends on configuration of the vehicle. In this paper complete 16 Degrees Of Freedom mathematical model of vehicle is considered for analysis which consists of powertrain(engine), chassis, four wheels as mass and engine mounts, suspensions and tyres as stiffness. This 16DOF mathematical model is solved using Wolfram Mathematica to find Natural frequencies and Mount displacements. The results obtained are validated with experimental results on actual system.

There are limitations on changing the mount locations frequently so, it becomes necessary to change rubber stiffness to meet desired NVH targets. Engine mount stiffness optimization is always a trade off between NVH performance and durability performance. Softer mounts are required to achieve NVH performance while harder mounts give more durability. In this work a optimization programme is developed which gives engine vibration and mount displacement from softer stiffness to harder one and find optimum value of engine mount stiffness using Wolfram Mathematica.

These learning's are useful for design of various subsystem or components to refine the full vehicle Noise Vibration
\end{abstract} Harshness (NVH) at the robust design level

Keywords -DSR, DEGREE OF FREEDOM (DOF), NATURAL FREQUENCY, VIBRATION, KINETIC ENERGY FRACTION.

\section{INTRODUCTION}

An engine which is perfectly balanced for forces and moments will have no tendency to move or to transmit vibration to the frame or foundation to which it is attached. Unfortunately, there is no such thing as a perfectly balanced engine. As a consequence, flexible mounts are needed for supporting an automobile engine and its peripheral components (e.g. gearbox). Among the reasons for using flexible mounts one may mention: to prevent the fatigue failure (caused by small dynamic disturbances) of the engine and gearbox support points; to reduce the amplitude of engine vibration which is transmitted to the vehicle's body structure; to reduce human discomfort and fatigue by partially isolating the engine vibrations from the body by means of an elastic media. Hence, the design of engine mounts must be performed with care in order to ensure that each and every one of the engine mount's functions can be accomplished successfully within some performance parameters ranges.

An engine mount system generally consists of three to four engine mounts. The behaviour of the mounting system not only depends on the performance of individual mounts but also on the complete system. The design of an engine mount system involves the selection of stiffness coefficients, location and orientations of the individual mounts. Traditionally, the mounts designed based on experience and extensive analysis procedures. Defining a near optimum initial mounting configuration is not an easy task due to the complex nature of the engine inertia properties and the packaging constraints on the mount locations as imposed by manufacturability considerations [1].

Yunhe Yu et al. [1] studied all types of engine mount and reported that though active engine mounts give good amount of vibration isolation, due to cost and weight they are not popular as elastomeric engine mounts. Kevin A. et al. [2] formulated the problem of vehicle modelling and ride quality optimization design within linear quadratic gausian control theory using kalman filter to identify vehicle parameters. John Bretl [3] determined response of driver seat hip using computerized optimization algorithm. D.S. sachdeva et al [4] compared three strategies all decoupled modes, coupled bounce-pitch modes and coupled bounce-roll modes to optimized to reduce vehicle response due to engine and road inputs. 
In this paper complete 16 Degrees Of Freedom mathematical model of vehicle is considered for analysis which consists of powertrain(engine), chassis, four wheels as mass and engine mounts, suspensions and tyres as stiffness. This 16DOF mathematical model is solved using Wolfram Mathematica to find Natural frequencies and Mount displacements. The results obtained are validated with experimental results on actual system.

\section{MATHEMATICAL MODELING}

For analytical analysis of any system first a mathematical model is required. Here also mathematical model for a vehicle system has been developed considering undammed lumped mass system.

\section{A. Natural frequency calculation by Eigen value analysis}

Calculation of natural frequencies of engine in case of 16 DOF model by Eigen value analysis is as follow.

General equation of motion is as follow

$$
[M] \ddot{x}+[K] x=0
$$

This is equation of motion of undammed free vibration and solution given by harmonic form as

$$
\mathrm{x}=\mathrm{X} \operatorname{Sin}(\omega \mathrm{t})
$$

Where

$\mathrm{X}=$ Amplitude.

$\omega=$ Circular natural frequency.

Substituting equation (2) in equation (1)

$-\omega^{2}[M] X \operatorname{Sin} \omega t+[K] X \operatorname{Sin} \omega t=0$

$\left.\left([\mathrm{K}]-\omega^{2}[M]\right)\{X\}\right)=0$

Pre multiplying above equation by $[M]^{-\mid}$will gate

$\left([M]^{-1}[K]-\omega^{2}[M]^{-1}[M]\right)\{X\}=0$

$[M]^{-1}[M]=I$

$$
\begin{gathered}
{[M]^{-\mid}[K]=[D]} \\
\left([D]-\omega^{2}[I]\right)\{X\}=0 \\
\operatorname{det}([D]-\lambda[I])=0 \\
\boldsymbol{f}_{\boldsymbol{l}}=\frac{\boldsymbol{\omega}_{\boldsymbol{l}}}{\mathbf{2 \pi}}
\end{gathered}
$$

Where,

$\lambda_{i}=\omega_{i}^{2}$

$f_{i}=$ Natural frequency 
$[\mathrm{M}]=$ Mass matrix of order $\mathrm{n} \times \mathrm{n}$

$[\mathrm{K}]=$ Stiffness matrix of order $\mathrm{n} \times \mathrm{n}$

$[D]=$ Dynamic matrix of order $n \times n$

A MATHEMATICA program is written to calculate natural frequencies, using $\mathrm{n}$ DOF equations of motion first then mass matrix [M] of size $n \times n$ and stiffness matrix $[K]$ of size $n \times n$ is formed compute dynamic matrix using equation (3) and $\mathrm{n}$ Eigen values $\lambda i$ of dynamic matrix which will give corresponding circular natural frequency and Eigen vectors will give mode shapes and Natural frequency in $\mathrm{Hz}$ can be determined by equation no (6).

\section{B. Kinetic Energy Fraction}

It is very important to identify modes of dynamic system as difficulty of mode identification of dynamic model increases substantially as model become larger and more complex.

A MATHEMATICA program is written to calculate natural frequencies as well as kinetic energy fraction, there are three types of output will be computed: Actual kinetic energy, Normalized kinetic energy and fractional kinetic energy.

1) Model kinetic energy is computed as follow :

$[$ Actual Kinetic energy $]=0.5 *\left(\left[M_{s}\right] .\left[U_{e}\right]\right) *\left[U_{e}\right]$

Where, $\left[M_{S}\right]$ is system mass matrix and $\left[U_{e}\right]$ is orthonormal mode shape matrix

2) The normalized energy is computed by normalizing each column of actual energy by the highest energy in each column.

3) The fractional energy is computed by dividing the normalized energy at given forcing frequency by sum of all normalized energies across all natural frequency.

Finally multiplying by 100 we will get \% kinetic energy matrix in which summation of all columns should be 100. From kinetic energy matrix we can assign natural frequencies to respective modes also we can observe coupling between two modes. Hence kinetic energy fraction matrix plays important role in mode identification.

\section{Mathematical Model}

Fig. 1 shows simple spring mass system 16 degree-of-freedom mathematical model which consists of 6 masses i.e. 4 wheels, chassis and engine. In this model we have considered 6 engine mounts, 4 suspensions and 4 tyres as stiffness. 


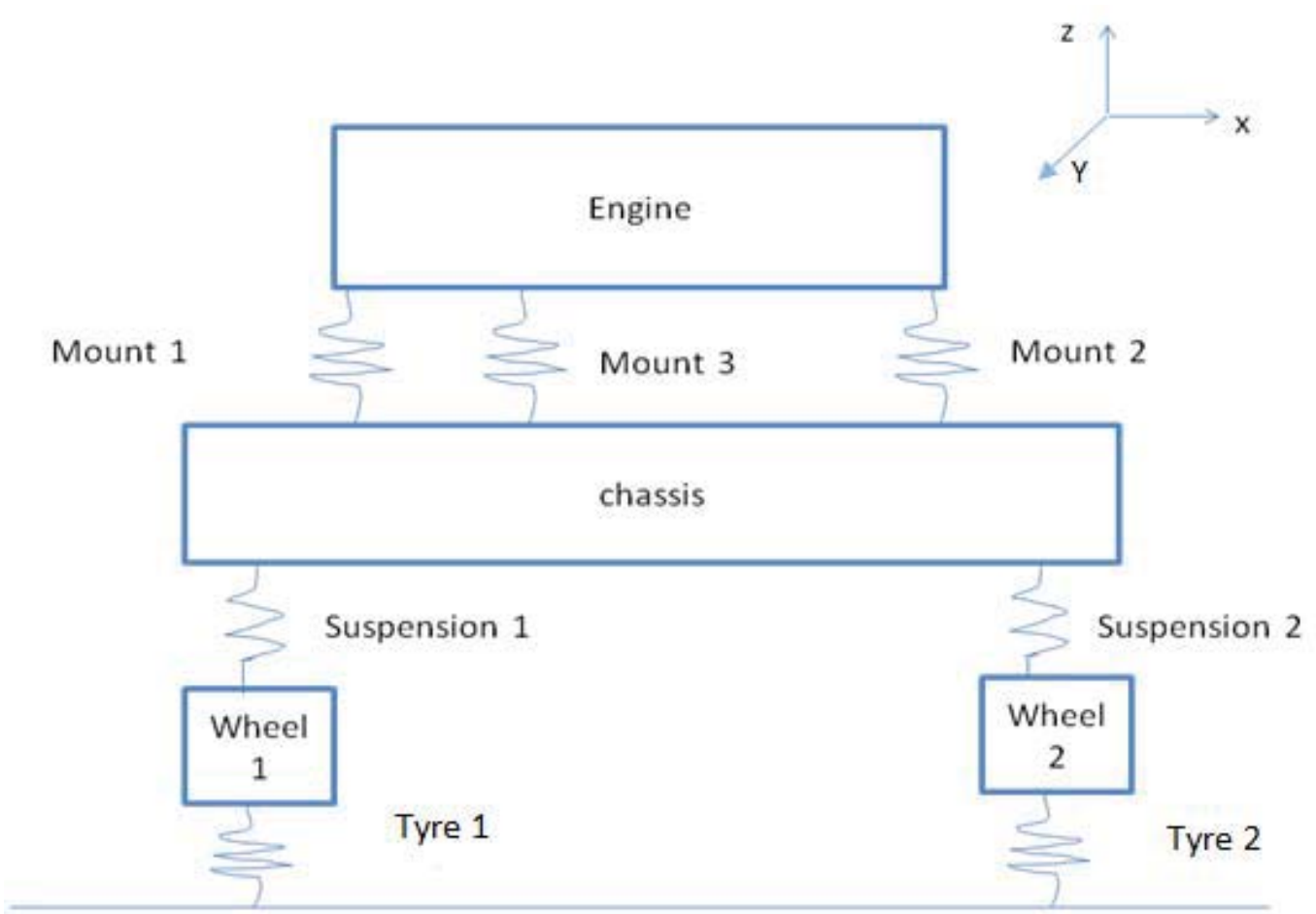

Fig. 116 DOF Mathematical model of vehicle.

Mass 1 (i.e. engine) is attached to mass 2 (i.e. chassis) through 6 springs (i.e. Engine mounts) and mass 2 (i.e. chassis) and mass 3,4,5 and 6 (i.e. four wheels) are connected through four springs (i.e. suspension) and finally mass 3,4,5 and 6 (i.e. four wheels) are connected to ground through 4 springs (i.e. tyres).In this mathematical model 16 DOF consider are as follows:

TABLE I: 16 DEGREES OF FREEDOM

\begin{tabular}{|l|l|c|l|}
\hline No. & \multicolumn{1}{|c|}{ Mass } & $\begin{array}{c}\text { Degree of } \\
\text { freedom } \\
\text { considered }\end{array}$ & \multicolumn{1}{c|}{ Directions } \\
\hline 1 & Wheel (FL) & 1 & Translation in $\mathbf{Z}$ direction only \\
\hline 2 & Wheel (RL) & 1 & Translation in $\mathbf{Z}$ direction only \\
\hline 3 & Wheel (RR) & 1 & Translation in $\mathbf{Z}$ direction only \\
\hline 4 & Wheel (FR) & 1 & Translation in $\mathbf{Z}$ direction only \\
\hline 5 & Chassis & 6 & Translation in $\mathbf{X}, \mathbf{Y}$ and $\mathbf{Z}$ directions \\
& & 6 & Rotational in $\mathbf{X}, \mathbf{Y}$ and $\mathbf{Z}$ directions \\
\hline 6 & Engine & & $\begin{array}{l}\text { Translation in } \mathbf{X}, \mathbf{Y} \text { and } \mathbf{Z} \text { directions } \\
\text { Rotational in } \mathbf{X}, \mathbf{Y} \text { and } \mathbf{Z} \text { directions }\end{array}$ \\
\hline
\end{tabular}

D. Equation of motions 
Equations of motion is derived using Newton's second law of motion which will give '16' second order differential equations of motion for ' 16 ' degree of freedom system. FBD of chassis and engine (powertrain) is considered to derive all equation of motions for mass 5 (chassis) and mass 6 (engine), where $Z_{5}$ and $Z_{6}$ are displacement of chassis and engine in $\mathrm{Z}$ direction and $\alpha_{5}$ and $\beta_{5}$ are rotational displacement of chassis about $\mathrm{X}$ and $\mathrm{Y}$ axis respectively and similarly $\alpha_{6}$ and $\beta_{6}$ are rotational displacement of engine about $\mathrm{X}$ and $\mathrm{Y}$ axis respectively.

Fig. 2 shows FBD of chassis and engine in Z-Y plane.

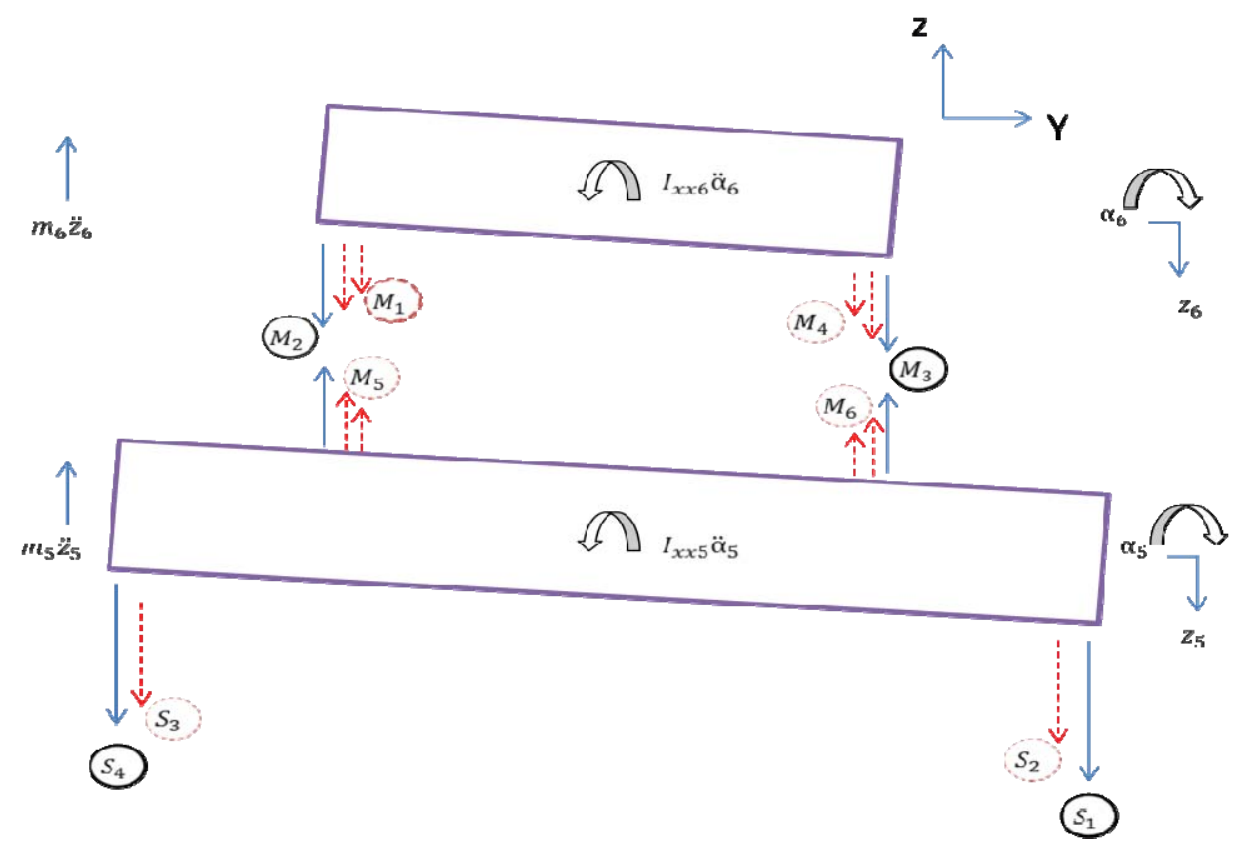

Fig.2 FBD of chassis and engine in Z-Y plane.

Translation of Engine in $\mathrm{X}$ direction.

$$
\begin{aligned}
& m_{6} \ddot{x}_{6}-x_{5}\left(\sum_{i=1}^{6} K x_{M i} * \operatorname{Cos} \psi i\right)+x_{6}\left(\sum_{i=1}^{6} K x_{M i} * \operatorname{Cos} \psi i\right)-\beta_{5}\left(\sum_{i=1}^{6} K x_{M i} * \operatorname{Cos} \psi i *\right. \\
& \left.M_{i z c}\right)+\beta_{6}\left(\sum_{i=1}^{6} K x_{M i} * \operatorname{Cos} \phi i * M_{i z p}\right)-\gamma_{5}\left(\sum_{i=1}^{6} K x_{M i} * \operatorname{Cos} \psi i * M_{i y c}\right)+ \\
& \gamma_{6}\left(\sum_{i=1}^{6} K x_{M i} * \operatorname{Cos} \psi i * M_{i y p}\right)=0
\end{aligned}
$$

Similarly all 12 equations of motion 6 for Engine and 6 for chassis are derived.

To derive remaining 4 equations of motion, system which only considers 3 masses i.e. wheel 1, wheel 2 and chassis is considered. Using FBD as shown in fig. 3 where $Z_{1}, Z_{2}, Z_{3}$ and $Z_{5}$ are displacement of wheel 1 , wheel 2 , wheel 3 and chassis in $Z$ direction and $\alpha_{5}$ and $\beta_{5}$ are rotational displacement of chassis about $\mathrm{X}$ and $\mathrm{Y}$ axis respectively we will derive equation of motion for mass 1 (wheel 1) and mass 2 (wheel 2) by Newton's second law of motion as follow.

Hence equation of motion for mass 1 (wheel 1) and mass 2 (wheel 2) are as follow:s 


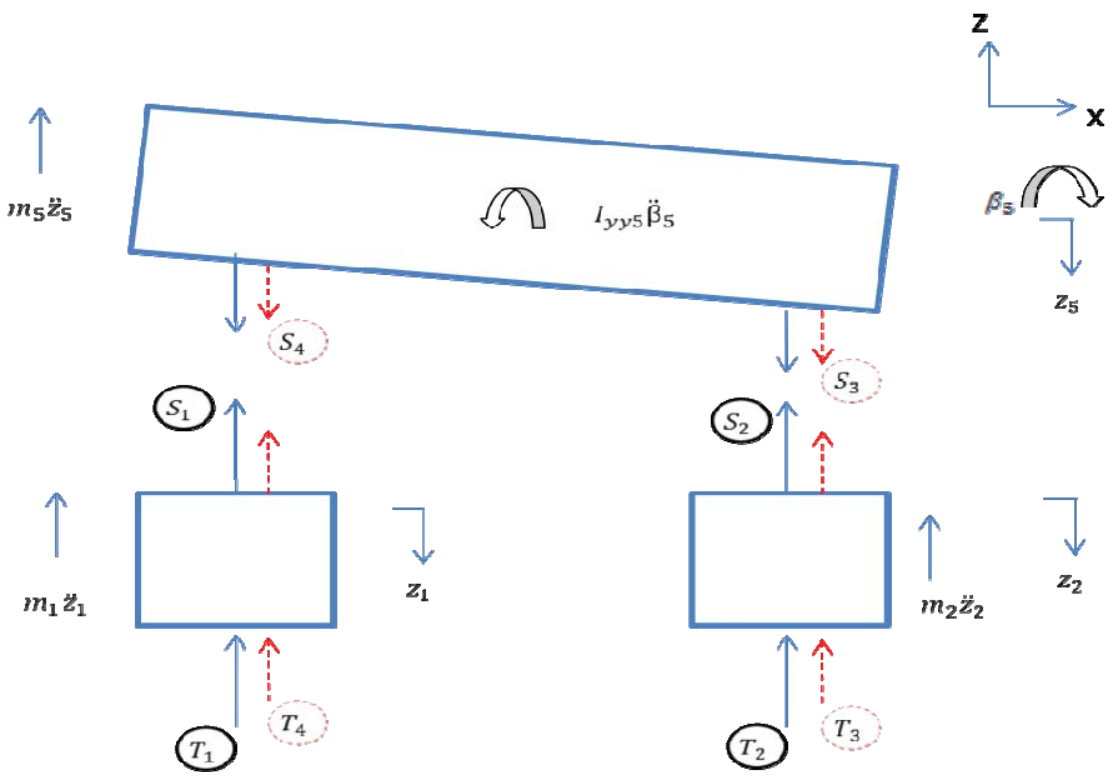

Fig.3 FBD considering wheel and chassis in Z-X plane.

Front left wheel (Displacement in Z direction only)

$$
m_{1} \ddot{Z}_{1}+\boldsymbol{Z}_{\mathbf{1}}\left(K z_{S 1}+K z_{T 1}\right)-\boldsymbol{Z}_{\mathbf{5}}\left(K z_{S 1}\right)-\boldsymbol{\beta}_{\mathbf{5}}\left(S_{1 x c} * K z_{S 1}\right)-\boldsymbol{\alpha}_{\mathbf{5}}\left(S_{1 y c} * K z_{S 1}\right)=0
$$

Rear left wheel (Displacement in Z direction only)

$$
m_{2} \ddot{Z}_{2}+\boldsymbol{Z}_{\mathbf{2}}\left(K z_{S 2}+K z_{T 2}\right)-\boldsymbol{Z}_{\mathbf{5}}\left(K z_{S 2}\right)-\boldsymbol{\beta}_{\mathbf{5}}\left(S_{2 x c} * K z_{S 2}\right)-\boldsymbol{\alpha}_{\mathbf{5}}\left(S_{2 y c} * K z_{S 2}\right)=0
$$

Similarly for mass 3 (wheel 3) and (wheel 4) we will derive equations.

\section{SOLUTION USING MATEMATICA PROGRAM}

Derived 16 equations of motion solved using MATHEMATICA script. A MATHEMATICA program is generated to calculate Natural frequency, \% Kinetic energy fraction and to determine Harmonic response of Driver's seat rail.

\section{A. Natural Frequency}

16 X 16 Mass Matrix [M] and 16 X 16 stiffness Matrix [K] is generated using MATHEMATICA program and Dynamic Matrix [D] is calculated as follow

$$
[D]=[M]^{-1}[K]
$$

Eigen values of matrix [D] will give natural frequency and Eigen vector of matrix [D] will give mode shapes.

\section{B. Kinetic Energy fraction}

The modal kinetic energy fraction is calculated to identify the significant local modes of subsystem for improving dynamic response prediction. KEF is measurement of amount of system kinetic energy contained within each subsystem for any given mode [8]. KEF is calculated as follow

\section{$[\mathrm{KEF}]=\frac{\left|\mathrm{U}_{\mathrm{c}}\right|^{\mathrm{T}}\left|\mathbf{M}_{\mathrm{c}}\right| *\left|\mathrm{U}_{\mathrm{c}}\right|}{\left[\mathrm{U}_{\mathrm{s}}\right]^{\mathrm{T}}\left[\mathrm{M}_{\mathrm{g}}\right] \cdot *\left[\mathrm{U}_{\mathrm{s}}\right]}$}

Where,

$\left[\mathrm{U}_{\mathrm{c}}\right]$ is component partition of the system mode shape matrix.

$\left[\mathrm{M}_{\mathrm{c}}\right]$ is component mass matrix. 
$\left(.^{*}\right)$ is element by element multiplication operator and element by element division operator is used for divison. C. Harmonic Analysis of Driver's seat rail (DSR)

Harmonic analysis of Driver's seat rail is done using mode superposition method and acceleration of Driver's seat rail in $\mathrm{z}$ direction at engine idling is computed using MATLAB program.

\section{RESULTS AND DISCUSSION}

MATHEMATICA program is solved using all input data for a 3 cylinder engine small car to calculate results. A. Natural Frequency and KEF

Following natural frequencies and KEF is obtained using MATLAB program for 3 cylinder engine small car.

TABLE II: 16 DOF NATURAL FREQUENCIES AND \%KEF

\begin{tabular}{|c|c|c|c|c|c|}
\hline Modes & $\begin{array}{c}\text { Natural Freq. } \\
(\mathrm{Hz})\end{array}$ & $\% \mathrm{KEF}$ & Modes & $\begin{array}{c}\text { Natural Freq. } \\
(\mathrm{Hz})\end{array}$ & $\% \mathrm{KEF}$ \\
\hline Engine f/a & 6.8 & 64.8 & $\begin{array}{l}\text { Chassis } \\
\text { vertical }\end{array}$ & 1.24 & 23.2 \\
\hline Engine lateral & 6.19 & 46.1 & Chassis roll & 1.44 & 71.6 \\
\hline Engine vertical & 8.39 & 54.5 & Chassis pitch & 1.93 & 51.6 \\
\hline Engine roll & 10.43 & 50.3 & Chassis yaw & 2.04 & 63.2 \\
\hline Engine pitch & 5.71 & 59.8 & $\begin{array}{c}\text { Wheel FL } \\
\text { vertical }\end{array}$ & 13.85 & 55 \\
\hline Engine yaw & 13.00 & 35.1 & $\begin{array}{c}\text { Wheel RL } \\
\text { vertical }\end{array}$ & 13.73 & 53 \\
\hline Chassis f/a & 1.47 & 45.8 & $\begin{array}{c}\text { Wheel RR } \\
\text { vertical }\end{array}$ & 13.73 & 53 \\
\hline Chassis lateral & 1.57 & 46 & $\begin{array}{c}\text { Wheel FR } \\
\text { vertical }\end{array}$ & 13.85 & 55 \\
\hline
\end{tabular}

All natural frequencies are within $20 \mathrm{~Hz}$. At idling condition i.e. at $850 \mathrm{rpm}$ frequency $14 \mathrm{~Hz}$, It is observed in table that all engine frequencies are away from $14 \mathrm{~Hz}$ so engine resonance will not occur. If any of the engine frequency is nearer to $14 \mathrm{~Hz}$ we need to change mount stiffness and mount location by trial and error method so that all engine frequencies will be away from $14 \mathrm{~Hz}$.

\section{B. Harmonic Analysis of Driver's seat rail}

Driver's seat rail acceleration of 3 cylinder small car is as shown in fig. 4 which is calculated solving 16 equations of motion by mode superposition method in MATHEMATICA program. 


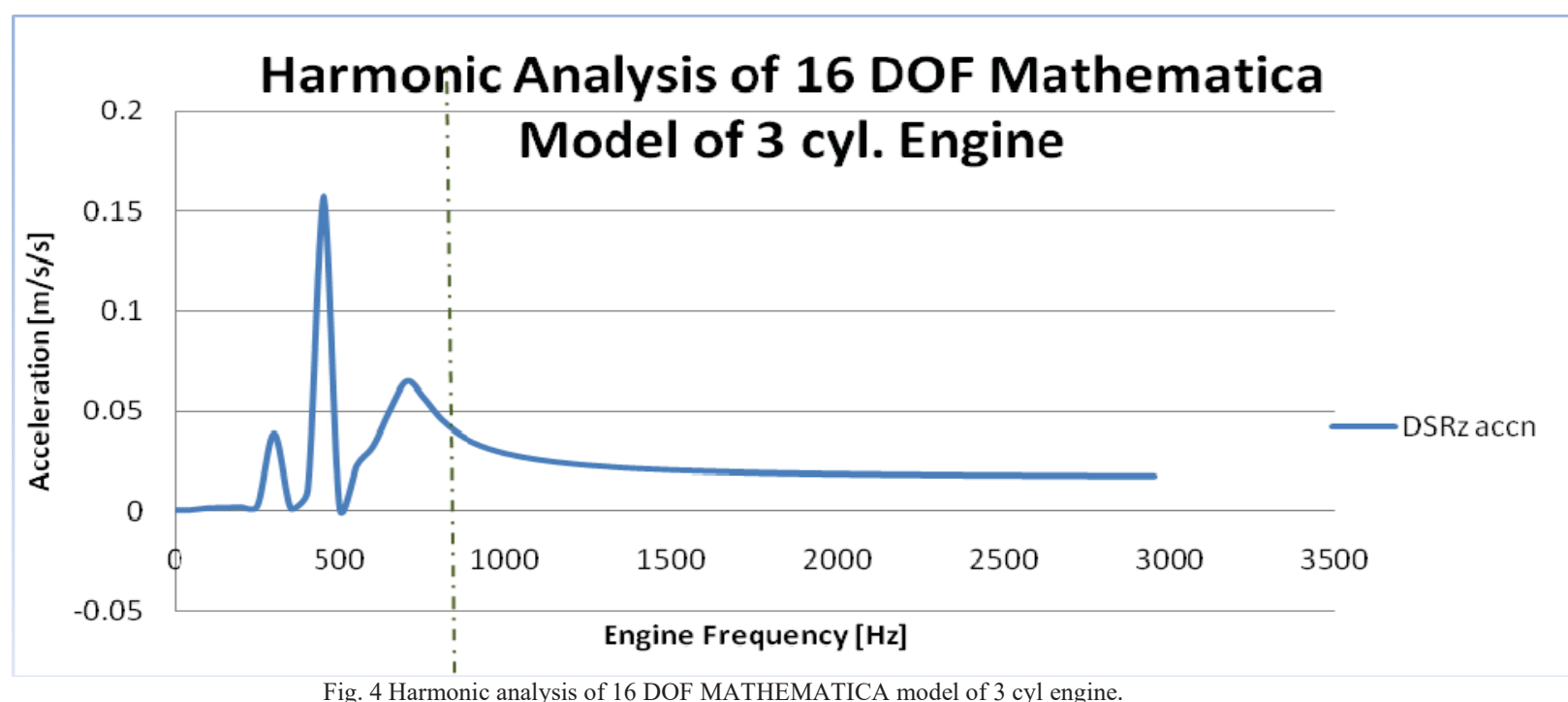

In fig. 4 we can see acceleration at idling i.e. at 850 Engine RPM is $39.12 \mathrm{~mm} / \mathrm{s} / \mathrm{s}$ which is near to $35 \mathrm{~mm} / \mathrm{s} / \mathrm{s}$ which is obtain experimentally using FFT analyzer and data acquisition system.

\section{CONCLUSION}

$\square$ A powerful MATHEMATICA tool is developed to determine natural frequencies, KEF of Engine.

Designer can predict vibrations of Driver's seat rail in initial design phase to predict mount stiffness and locations.

$\square$ Hence further multidisciplinary optimization required to predict correct mount stiffness and mount locations in initial design stage.

\section{ACKNOWLEDGMENT}

Thanks Dr. Milind Ambardekar, Mr. Dilip Sahu and Prasad Kulkarni from TATA MOTORS LTD for their valuable guidance in this work.

\section{REFERENCES}

[1] Kevin A. Wise, Robert E. Reid, Modeling and identification of a light Truck engine mounting system for ride quality optimization, SAE paper 841142, 1984

[2] John Bretl, Optimization of engine mounting systems to minimize vehicle vibration, SAE paper 931322, 1993.

[3] Yunhe Yu, Nagi G. Naganathan, A literature review of automotive vehicle engine mounting systems, Rao Dukkipati, mechanisum and machine theory 36 (2001) pp.123-142

[4] D.S.Sachdeva,R.Hadi, Effect of engine mounting strategy on vehicle NVH, SAE paper 2003-01-1467.Mohammad Sirafi, Mohamad Qatu, Accurate Modeling for the Powertrain and Subframe Modes, SAE paper 2003-01-1469.

[5] Magnus Olsson, Mikael Törmänen, Sylvain Sauvage and Catharina Hansen, Systematic multi-disciplinary optimization of engine mount, SAE paper 2011-01-1674.

[6] Cyirl M. Harris, Harri's shock and vibration handbook, 5th edition, McGraow Hill, 2002, pp. 2.1-3.56

[7] Y.T.Chung, L.L. Kahre, A general procedure for finite element model check and model identification

[8] Kwon-Hee suh, A study on balancing of the 3 cylinder engine with balance shaft, SAE 2000-010601

[9] Jianghua Fu1, Jian Pang ; Chengtai Hu1; Xiaomin Xu1, Renwei Deng Xiaohong Kuang, Study on startup transient vibration of a vehicle with 3-cylinder engine, inter.noise 2014 melbourn, austrelia 